\title{
Planck, Dieter, Thiel, Andreas, Das Limes-Lexikon. Roms Grenzen von A bis $Z$
}

\section{Thomas Lienhard}

\section{OpenEdition}

\section{Journals}

Édition électronique

URL : http://journals.openedition.org/ifha/2125

DOI : $10.4000 /$ ifha. 2125

ISSN : 2198-8943

\section{Éditeur}

IFRA - Institut franco-allemand (sciences historiques et sociales)

Référence électronique

Thomas Lienhard, «Planck, Dieter, Thiel, Andreas, Das Limes-Lexikon. Roms Grenzen von A bis Z », Revue de l'IFHA [En ligne], Date de recension, mis en ligne le 01 janvier 2010, consulté le 22 septembre 2020. URL : http://journals.openedition.org/ifha/2125 ; DOI : https://doi.org/10.4000/ifha.2125

Ce document a été généré automatiquement le 22 septembre 2020.

(CIFHA 


\title{
Planck, Dieter, Thiel, Andreas, Das Limes-Lexikon. Roms Grenzen von A bis Z
}

\author{
Thomas Lienhard
}

1 Écrivons-le d'emblée : ce livre ne répond pas, et ne cherche peut-être pas à répondre aux exigences d'une présentation scientifique. Il est vrai que ce parti pris peut parfaitement se justifier étant donné le cadre éditorial dans lequel s'inscrit l'ouvrage : la collection "Beck'sche Reihe », qui fut déjà souvent présentée dans notre Revue, s'adresse à un public plus large que celui des seuls scientifiques, à l'instar des "Que sais-je ? » français. Mais dans la mesure où la présente recension s'adresse, elle, aux chercheurs, il importe de souligner quelques points afin d'éviter tout malentendu.

Une première lacune dont souffriront les scientifiques face à ce dictionnaire porte sur l'imprécision des références. Certes, les articles sont (généralement) accompagnés d'une brève bibliographie ; mais celle-ci est, dans l'ensemble, relativement ancienne. Ce point permet d'ailleurs d'expliquer quelques bévues telles que l'emploi de toponymes germaniques pour des espaces qui ne le sont plus depuis bien longtemps. Ainsi, l'auteur de la notice sur Straßburg (soit...) mentionne, pour décrire le tracé du camp romain dans cette ville, le nom de la Spiesgasse et de la Münstergasse, présentés comme des repères actuels : l'auteur des présentes lignes le met au défi de retrouver aujourd'hui ces noms dans les rues de Strasbourg. Par ailleurs, les sources historiques ne sont pratiquement jamais mentionnées ou, dans le cas contraire, ne le sont pas avec une précision suffisante pour permettre au lecteur intéressé de retrouver telle ou telle occurrence. De même, les illustrations, souvent parfaitement choisies, semblent avoir une fonction essentiellement décorative, sans que le commentaire ne permette de les identifier avec exactitude. Dans ces divers domaines, les éditeurs de l'ouvrage ont manifestement sacrifié la précision à la concision.

3 Une autre lacune, qui ne frustrera pas seulement les scientifiques, réside dans l'absence d'une quelconque introduction. La conséquence immédiate en est qu'il sera difficile au lecteur de se faire une idée, sur la base de ce seul livre, à propos de la nature exacte du 
limes (alors qu'une mise au point historiographique à propos d'un sujet aussi polémique n'aurait pourtant pas été superflue) : s'agissait-il d'une barrière linéaire ? D'un chapelet de fortifications ponctuelles ? D'un espace-frontière dilaté sur une certaine profondeur géographique ? D'un concept purement administratif, qui n'était pas toujours incarné dans le paysage ? Certes, on trouvera une (fort brève) entrée " limes " au sein même du dictionnaire, mais d'une part il est curieux de retrouver cantonné au rang de notice ce qui constitue en réalité le sujet du livre, et d'autre part, elle ne peut en aucun cas remplacer une introduction : si elle fournit une petite analyse des significations antiques pour limes, elle ne propose aucune synthèse à propos de la manière dont se présente réellement cette structure immense dans la perspective de l'historien ou de l'archéologue. Au sortir de la lecture, on éprouve donc la sensation d'avoir collecté des informations parfois remarquables mais éparses, sans avoir compris ce qu'était effectivement le limes dans son ensemble.

4 L'absence d'introduction empêche également de savoir sur quelle base fut effectué le choix des notices. Le lecteur doit donc se faire lui-même une idée, au fil de la lecture, à propos de ce qu'il peut espérer trouver, ce qui relève parfois de la tombola documentaire dans le cas d'un ouvrage aussi bref. Pour lui éviter ce fastidieux travail de reconstitution, signalons ici que dans ce dictionnaire, la géographie du limes, son architecture et les éléments techniques de la vie militaire ont la part belle et que les thèmes religieux liés à la guerre sont bien représentés également ; en revanche, les responsables éditoriaux ont renoncé à pousser plus loin l'élargissement thématique, ce qui aurait pu aboutir à une histoire totale du sujet considéré. Ce parti pris a l'inconvénient de sacrifier des thèmes importants comme la vie sociale dans l'armée, le commerce autour des fortifications, ou encore la perception de la frontière ou de l'étranger par les Romains ; signalons également que l'ensemble des notices est très germano-centré, n'accordant que deux entrées en tout à l'Orient ou à l'Afrique.

Cela dit, l'avantage incontestable de ce choix éditorial réside dans la précision des articles qui, à défaut de présenter des thèmes généraux, font bien le point sur les sujets effectivement retenus : celui qui s'intéresse aux grands sites archéologiques allemands liés au limes, à l'armement ou à la hiérarchie militaire sera ici comblé. Par ailleurs, et même si le choix des entrées est parfois frustrant, il comporte également quelques heureuses trouvailles. Ainsi, à défaut d'une présentation historiographique pour chaque thème abordé, le lecteur trouvera ici plusieurs notices sur d'importants historiens contemporains qui ont contribué à élaborer notre perception du système défensif romain. De même, les auteurs ont pensé à fournir quelques éléments concernant la postérité du limes pour les périodes durant lesquelles celui-ci ne constitua plus un ensemble militaire cohérent : outre l'article "Limesfall ", qui relativise la rapidité de cette évolution, l'entrée "Teufelsmauer " apporte bien des surprises à propos des croyances qui, à l'époque moderne, se virent associées avec l'antique chaîne défensive que constituait le limes. On l'aura compris : ce livre a une présentation moins scientifique qu'un ouvrage de recherche (et également que d'autres numéros de la belle collection dans laquelle il est paru), mais il rendra des services tout de même.

Thomas Lienhard (IFHA) 